\title{
Structure of Disordered Eating in a Twin Community Sample
}

\author{
Tracey Wade \\ Marika Tiggemann \\ Andrew C. Heath \\ Suzanne Abraham \\ Susan A. Treloar \\ Nicholas G. Martin \\ (Accepted 6 March 1995)
}

\begin{abstract}
Objective: To describe the types of eating problems experienced by women in an Australian twin population. Method: Questions assessing preoccupation with weight or shape, use of various methods of weight control, difficulties with weight control, disordered eating, or binging, were administered to a group of 3,869 female twins. Exploratory and confirmatory factor analyses were used to summarize and describe problematic eating behaviors. Results: For those women experiencing problems with eating, five groups could be identified. These were overweight women who were dissatisfied with their weight and shape, underweight women struggling with anorexic behaviors, women who were having problems with binging, women who were using more extreme methods of weight control such as vomiting, laxatives, and starvation, and overweight women who were using slimming and fluid tablets for weight control. Confirmatory factor analysis suggested that this factor model is an acceptable fit to the data and that the factor structure generalizes well across two groups viz, the first-born and second-born twins. Discussion: It was concluded that future studies aiming to develop a general description of eating problems in the community should specifically assess the purging behaviors used by women. (c) 1996 by John Wiley \& Sons, Inc.
\end{abstract}

Over the last 10 years the diagnostic definitions of bulimia nervosa and anorexia nervosa have been progressively modified in order to present a more specific and restrictive

\footnotetext{
Tracey Wade is a Ph.D. candidate from the School of Psychology, Flinders University of South Australia; Marika Tiggemann, Ph.D., is Senior Lecturer in Psychology at the Flinders University of South Australia; Andrew C. Heath, D.Phil., is Professor with the Department of Psychiatry, Washington University Medical School, St. Louis, Missouri; Suzanne Abraham, Ph.D., is Associate Professor with the Department of Obstetrics and Gynaecology, University of Sydney; Susan A. Treloar, Ph.D., is Research Associate with the Queensland Institute of Epidemiology; and Nicholas Martin, Ph.D., is Professor of Epidemiology with the Queensland Institute of Medical Research. Address all correspondence to Tracey Wade, School of Psychology, Flinders University of South Australia, GPO Box 2100, Adelaide, 5001, Australia.
} 
description. Within the same population, the earlier diagnostic definition of the Diagnostic and statistical manual of mental disorders' (3rd ed., DSM-III) bulimia nervosa (American Psychiatric Association, 1980) can be up to seven times higher than the more recent manual's 3rd rev. ed.'s, (DSM-III-R) diagnosis (American Psychiatric Association, 1987). The manual's 4th ed.'s (DSM-IV; American Psychiatric Association, 1994) diagnostic criteria further acknowledge this specificity, developing an additional definition of Eating Disorder Not Otherwise Specified (EDNOS) to permit the recognition of the variety of eating problems that do exist in the general community apart from bulimia nervosa and anorexia nervosa. These eating problems cover a wide range, including women who meet criteria for anorexia nervosa except that they continue to have regular menses, people who meet all of the criteria for bulimia nervosa but who binge less than twice a week, individuals of normal body weight who regularly attempt to compensate for the intake of small amounts of food, and individuals who recurrently binge eat but who do not attempt to purge. It is estimated that about $10 \%$ of the population have experienced some disordered eating (Wade et al., 1994), which is about five times as many people who develop either bulimia nervosa or anorexia nervosa.

To date, only a few studies have examined the structure of eating problems in the general population. The usual method of investigation is to use exploratory factor analysis to summarize information on eating habits and attitudes using latent factors that are interpreted in relation to the content of the observed variables. Some studies have confined themselves to measuring only eating attitudes rather than behavior (Ben-Tovim \& Walker, 1992). Of the three studies found in the literature that look at disordered eating behavior in the general community, one has used an already existing measure of eating pathology (Lavik, Clausen, \& Pedersen, 1991) and two have constructed new scales for the purpose (Coker \& Roger, 1990; Mehrabian \& Riccioni, 1986). Two of these studies have found a similar factor structure, involving three factors: dieting, restrained eating patterns/anorexia, and overeating/bulimia (Lavik et al., 1991; Coker \& Roger, 1990). However, apart from dietary restraint, none of these studies measure methods of weight control such as vomiting, excessive exercise, or abuse of laxatives, which are common accompaniments to bulimia nervosa and are behaviors that up to a third of the population have experienced at some stage in their life (Wade et al., 1994).

The aim of the present study is to describe the eating behavior of a large Australian twin sample by factor analysis of a self-report questionnaire, assessing both attitudes and behavior as they relate to preoccupation with weight or shape, use of various methods of weight control, difficulties with weight control, disordered eating, and binging.

\section{METHOD}

\section{Subjects}

In 1988-1989 a battery of self-report questionnaires measuring a wide variety of psychological, social, demographic, and health characteristics was sent to twin pairs registered with the Australian National Health and Medical Research Council Twin Register (Heath, Cloninger, \& Martin, 1994). Only data from the 3,869 females who completed the questions relating to eating were examined. The age range of this group was between 24 and 86 years, the mean age (on January 1,1989$)$ was 41.9 years $(S D=13.1$ ). An examination of the sociodemographic features of the female questionnaire respon- 
dents (Treloar, 1992), including age, marital status, educational background, workforce participation, major lifetime occupation, and religious denomination suggests that the sample is not notably different from the Australian female population (using data obtained from the Australian Bureau of Statistics between 1986 and 1992).

\section{Assessment of Eating}

Eating problems were assessed by five items in the questionnaire battery, all of which required a yes or no answer. For questions 1-4, subjects were asked to report both "now" or "previously."

Question 1. Do you feel that you have difficulty controlling weight?

Question 2. Do you feel you have had problems with disordered eating?

Question 3. Have you ever used any of the following methods to control your body weight-starvation, self-induced vomiting, excessive exercise, laxatives, fluid tablets, or slimming tablets?

Question 4. Do you feel you have been preoccupied with thoughts of food or body weight?

Question 5. Have you ever suffered from or been treated for an eating disorder, low body weight, binge eating, obesity, weight loss, anorexia nervosa, or bulimia?

These questions produced a total of 16 variables concerning eating problems which were entered into an exploratory principal components factor analysis so that the structure of eating difficulties in a large community sample could be examined.

\section{Methods of Analysis}

Initially, the eating questions were examined using exploratory factor analysis. In order to test the goodness of fit of the model suggested by the exploratory factor analysis, confirmatory factor analysis (CFA) was carried out using Lisrel for Windows 8.03 and Prelis 2.03 (Joreskog \& Sorbom, 1993). In exploratory factor analysis, the researcher has limited control over the structure to be tested and passively interprets the empirical results in relation to theory and prior research. In CFA the researcher explicitly posits one or more a priori models from exploratory factor analysis, fits the a priori model to the observed data, evaluates the goodness of fit of the model, and modifies the model (a posteriori models) so as to improve its parsimony and/or its fit to the data (Marsh, 1991; MacCallum, 1986).

\section{RESULTS}

\section{Exploratory Factor Analysis of Eating Variables}

Exploratory factor analysis was carried out with the questionnaire items relating to eating problems. Varimax rotation was implemented to facilitate interpretation of factors. This resulted in five factors with eignevalues greater than 1 , together accounting for $58.9 \%$ of the variance. These results are summarized in Table 1, with factor loadings greater than 0.3 in bold. 
Table 1. Factor loadings $(\times 100)$ for 16 disordered eating variables $(N=3,030$ individuals), using varimax rotation with principal components analysis

\begin{tabular}{lrrrrr}
\hline \multicolumn{1}{c}{ Variables } & Factor 1 & Factor 2 & Factor 3 & Factor 4 & Factor 5 \\
\hline Difficulty with weight control & $\mathbf{7 6}$ & -03 & -00 & 10 & 16 \\
Preoccupation with weight & $\mathbf{7 0}$ & 07 & 03 & $\mathbf{3 0}$ & 12 \\
Disordered eating & $\mathbf{7 0}$ & 15 & 12 & 24 & 06 \\
Obesity & $\mathbf{5 0}$ & 05 & $\mathbf{3 0}$ & -28 & 32 \\
Low body weight & -01 & 84 & -03 & 06 & -02 \\
Weight loss & 08 & 83 & -03 & 00 & 06 \\
Anorexia nervosa & 01 & 60 & 24 & 17 & -01 \\
Eating disorder & 21 & 53 & 42 & 00 & -01 \\
Bulimia & -06 & 10 & 80 & 16 & 06 \\
Binging & 22 & 09 & 76 & -00 & 00 \\
Starvation & 24 & 16 & 10 & 64 & 13 \\
Excessive exercise & 31 & 05 & -01 & 62 & -04 \\
Laxatives & 05 & 05 & 09 & 55 & $\mathbf{5 0}$ \\
Vomiting & -05 & 02 & $\mathbf{5 1}$ & 55 & 08 \\
Fluid tablets & 09 & 02 & 05 & 03 & 84 \\
Slimming tablets & 37 & -04 & -02 & 15 & 65 \\
Eigenvalues & 3.8 & 2.0 & 1.4 & 1.2 & 1.0 \\
\% age of variance & 23.8 & 12.7 & 8.8 & 7.2 & 6.4 \\
\hline
\end{tabular}

Note. Factor loadings equal to or greater than 0.30 are in bold.

Factor I, called an overweight factor, loaded primarily on problems with eating and being overweight and includes: ever experiencing difficulty with controlling weight, ever experiencing a preoccupation with weight or eating, ever suffering from or being treated for disordered eating or obesity. Factor II, an anorexia factor, loaded on problems with low weight, including ever suffering from or being treated for low body weight, weight loss, anorexia nervosa, or an eating disorder. Factor III is clearly a bulimia factor including ever suffering from or being treated for bulimia and binge eating. Ever having an eating disorder and use of self-induced vomiting as a means of weight control also had a substantial loading on this factor. Factor IV, the first of two purging factors, is an extreme weight control methods factor, involving ever using starvation, excessive exercise, laxatives, and vomiting for weight control. The final factor, Factor V, is the second tablet purging factor and includes use of fluid or slimming tablets for weight control.

\section{Relationship Between Factor Scores, Body Mass Index (BMI), and Demographic Variables}

In order to see if the factor scores were differentially affected by BMI, age, education levels, and self-assessed social class, Pearson correlations were calculated. The education level was assessed by asking respondents about the highest education level completed. Responses were rated on a 7-point scale, beginning at less than 7 years' schooling and ending in university postgraduate training. Social class was assessed by asking the question, "If you were asked to describe your social class, which of these items would you use?" Respondents were asked to choose one of three categories, working class, middle class, or upper class.

To counter the possibility of Type I errors, only correlations significant at the 0.01 level are highlighted (see Table 2). BMI was significantly higher for the overweight and tablet purging factor scores, and significantly lower for the anorexia and extreme methods of weight control factor scores. In terms of the demographic variables, the women experiencing more problems with the overweight factor behaviors were younger. Those 
women who had more problems with the behaviors associated with the bulimia factor had completed more years of education. The extreme methods of weight control factor were associated with being younger and having more years of education. The tablet purging behaviors were associated with less years of education and lower class.

\section{CFA}

Traditionally, when evaluating goodness of fit in CFA, the chi square test for statistical significance has been used, whereby a nonsignificant chi square is interpreted to mean that the model fits the data (Marsh \& Smith, 1987). However, it is now recognized that this is an inadequate test of fit and alternative indicators of fit have been developed. The Tucker-Lewis index or nonnormed fit index (NNFI) (Tucker \& Lewis, 1973) is relatively free from sample size contamination and imposes an appropriate penalty function for the inclusion of additional parameters (Ferguson, Dodds, Ng, \& Flannigan, 1994; Marsh, 1990). The comparative fit index (CFI; Bentler, 1990) is an unbiased counterpart of the fit index originally proposed by Bentler and Bonett (1980). The CFI evaluates the adequacy of the specified model in relation to the baseline model, or null model, which specifies no relationship amongst the observed variables (i.e., every measure is an indicator for a separate latent variable) (Feldman, 1993). For both the NNFI and the CFI the fit coefficients range from 0 to 1 with higher values indicating greater fit; a value of 0.9 or greater is accepted to indicate that the hypothetical model fits the data well (Feldman, 1993; Marsh, 1991).

The model that was tested for goodness of fit consisted of five factors, including all factor loadings greater than or equal to 0.3 , as seen in Table 1 . This a priori model had a CFI of 0.89 and a NNFI of 0.85 and is therefore not fully adequate. Using the modification indices suggested by LISREL, the addition of the "ever had difficulty controlling weight" and "ever suffered from or been treated for anorexia nervosa" items to the bulimia factor increased fit to a satisfactory level $(C F I=0.91 ; N N F I=0.87)$. Given that the a priori model is shown to have a fairly good fit to the data and did not require much adjustment to create a satisfactory fit, it was felt that the factor scores from the a priori model would be suitable to be used in further analyses.

\section{Generalizability of the Factor Structure}

Although it is often not possible to cross-validate findings of CFA, it is highly recommended as it provides a test of the generalizability of the a priori solutions and may control for capitalization on chance when testing a posteriori models (Marsh, 1991). The

Table 2. Pearson correlations $(\times 100)$ between factor scores and body mass index (BMI), age, education, class

\begin{tabular}{lccccc}
\hline Variable & $\begin{array}{c}\text { Factor } \\
\text { Score I }\end{array}$ & $\begin{array}{c}\text { Factor } \\
\text { Score II }\end{array}$ & $\begin{array}{c}\text { Factor } \\
\text { Score III }\end{array}$ & $\begin{array}{c}\text { Factor } \\
\text { Score IV }\end{array}$ & $\begin{array}{c}\text { Factor } \\
\text { Score V }\end{array}$ \\
\hline BMI & $22^{*}$ & $-07^{*}$ & 02 & $-06^{*}$ & $10^{*}$ \\
Age & $-13^{*}$ & -02 & -02 & $-18^{*}$ & 03 \\
Education & 01 & 03 & $05^{*}$ & $05^{*}$ & $-08^{*}$ \\
Class & -03 & -02 & 02 & 01 & $-06^{*}$ \\
\hline
\end{tabular}

*Pearson correlations significant at the .01 level. 
twin design is an ideal vehicle for testing such generalizability as it has built-in ability, because of its inherent matched pair nature, to test factor similarity.

The first-born twins and their cotwins were entered separately as subsamples (twin 1 and twin 2 subsamples) into a principal factor analysis using varimax rotation. The twin 1 analysis resulted in the extraction of five factors accounting for $60.4 \%$ of the variance and the twin 2 analysis resulted in the extraction of five factors accounting for $58.0 \%$ of the variance (see Table 3: factor loadings greater than 0.3 are selected only).

In the twin 1 (T1) analysis, there are still basically the same five factors, the most notable exceptions being that vomiting is now most strongly associated with the anorexia factor. In the twin 2 (T2) analysis, there are once again basically the same five factors as the overall analysis except that the eigenvalue and percent of variance accounted for by the extreme weight control methods factor are larger than for the bulimia factor.

The first test of the equivalence of the factor structure between the two populations was calculated using the Tucker congruency coefficient (Tucker, 1951). The coefficients for the five factors range from 0.93 to 0.98 , indicating that the factors are highly similar between the two populations. Although there are no tests of significance associated with the coefficients of congruence, coefficients of 0.90 are generally accepted as indicating a highly stable structure (Tobin, Holroyd, Reynolds, \& Wigal, 1989).

A second test of the equality of factor structures, and one that allows some identification of the weaknesses of the model, examines the ability of the hypothesized factor structure to fit responses from each group without requiring any parameter estimates to be the same across groups. The three parameter estimates of interest are the error variance (EV), referring to the EV for each observed variable, the factor correlation (FC), that is the correlations between latent variables, and the factor loadings (FL) which

Table 3. Factor loadings $(\times 100)$ for first-born twin $(\mathrm{T} 1)$ and second-born twin $(\mathrm{T} 2)$ subsamples, using varimax rotation with principal components analysis

\begin{tabular}{|c|c|c|c|c|c|c|c|c|c|c|}
\hline \multirow[b]{2}{*}{ Variables } & \multicolumn{2}{|c|}{$\begin{array}{l}\text { Overweight } \\
\text { Factor }\end{array}$} & \multicolumn{2}{|c|}{$\begin{array}{l}\text { Anorexia } \\
\text { Factor }\end{array}$} & \multicolumn{2}{|c|}{$\begin{array}{l}\text { Bulimia } \\
\text { Factor }\end{array}$} & \multicolumn{2}{|c|}{$\begin{array}{c}\text { Extreme } \\
\text { Weight Loss } \\
\text { Factor }\end{array}$} & \multicolumn{2}{|c|}{$\begin{array}{l}\text { Tablet } \\
\text { Purging } \\
\text { Factor }\end{array}$} \\
\hline & $\mathrm{T} 1$ & T2 & T1 & T2 & $\mathrm{T} 1$ & $\mathrm{~T} 2$ & $\mathrm{~T} 1$ & $\mathrm{~T} 2$ & $\mathrm{~T} 1$ & $\mathrm{~T} 2$ \\
\hline Weight control & 74 & 79 & & & & & & & & \\
\hline Preoccupation & 66 & 73 & & & & & & 36 & & \\
\hline Disordered eating & 67 & 70 & & & & & & & & \\
\hline Obesity & 57 & 45 & & & & 41 & & -30 & 39 & 37 \\
\hline Low body weight & & & 84 & 84 & & & & & & \\
\hline Weight loss & & & 83 & 82 & & & & & & \\
\hline Anorexia nervosa & & & 68 & 49 & 33 & & & & & \\
\hline Eating disorder & & & 45 & 61 & 48 & 36 & & & & \\
\hline Bulimia & & & & & 82 & 79 & & & & \\
\hline Binging & 30 & & & & 74 & 78 & & & & \\
\hline Starvation & & & & & & & 67 & 64 & & \\
\hline Excessive exercise & & & & & & & 58 & 67 & & \\
\hline Laxatives & & & & & & & 57 & 52 & 54 & 48 \\
\hline Vomiting & & & 67 & & 62 & 30 & 61 & 50 & & \\
\hline Fluid tablets & & & & & & & & & 84 & 81 \\
\hline Slimming tablets & 41 & 34 & & & & & & & 61 & 67 \\
\hline Eigenvalues & 3.8 & 3.8 & 2.1 & 1.9 & 1.5 & 1.1 & 1.2 & 1.4 & 1.0 & 1.0 \\
\hline$\%$ age of variance & 24.0 & 23.8 & 13.3 & 12.1 & 9.1 & 7.0 & 7.7 & 8.8 & 6.4 & 6.3 \\
\hline
\end{tabular}

Note. Factor loadings equal to or greater than 0.30 are included only. 
indicate the degree of relationship between a latent factor and an observed variable. A series of increasingly restrictive models are then fitted to the data and this allows us to both identify the parameters that are most responsible for differences between different populations (Joreskog \& Sorbom, 1993), and to ensure that a totally invariant model (i.e., all parameters set to be equal across populations) is not very different in terms of fit statistics from the totally free model where parameters are allowed to be different across populations (Marsh \& Smith, 1987). Results of model fitting in this manner are summarized in Table 4.

Model 5 which constrains all parameters to be the same differs from model 1 in a strict statistical sense but the goodness of fit indices for models 1 to 5 are much the same, indicating that factor structure is similar across responses by twin 1 and twin 2 subsamples. Examination of the difference in chi square from model 1 to the other models indicates that the inclusion of the factor correlations causes most of the difference between the models. Closer examination of the factor correlations between the two groups indicates that it is mainly the correlation of the bulimia factor and the first purging factor to the other factors that are responsible for the differences, suggesting that the bulimia factor and the first purging factor are the least stable of the factors.

\section{DISCUSSION}

\section{Structure of Disordered Eating}

Using measures of self-reported eating behavior from a large representative sample of women, five factors were identified that described the problems experienced by this population. The first factor, identified by exploratory factor analysis, was an overweight factor. This factor is associated with experiencing difficulties with weight control, a preoccupation with food or weight, encountering problems with disordered eating, and perceiving oneself to have problems with obesity. The weight loss methods of excessive exercise and slimming tablets are also associated with this factor. This first factor is somewhat similar to the dieting factor found by Lavik and colleagues (1991) and Coker and Roger (1990), in that it contains preoccupation with weight and food and dissatisfaction with body shape. However, it appears to focus more on younger women who have experienced problems with being overweight (i.e., a higher BMI) rather than on

Table 4. Goodness-of-fit indices for modeis testing factorial equality across twin 1 and twin 2 populations

\begin{tabular}{|c|c|c|c|c|}
\hline Model & $x^{2}(\mathrm{df})$ & $x^{2}$ diffa & $\mathrm{CFI}$ & NNFI \\
\hline 1: no invariance ${ }^{b}$ & $1,779(172)$ & - & 0.89 & 0.85 \\
\hline 2: FL invariant & $1,886(196)$ & 107 & 0.89 & 0.86 \\
\hline 3: $F L$ and $F C$ invariant & $1,935(206)$ & 156 & 0.89 & 0.87 \\
\hline 4: $\mathrm{FL}$ and $\mathrm{EV}$ invariant & $1,911(212)$ & 132 & 0.89 & 0.87 \\
\hline 5: Total invariance & $1,963(222)$ & 184 & 0.88 & 0.88 \\
\hline
\end{tabular}

Note. $\mathrm{CFI}=$ comparative fit index; $\mathrm{NNFI}=$ nonnormed fit index.

${ }^{2} x^{2}$ diff refers to the difference between the chi square of the a priori model and the model in question.

bFactor loadings (FL), factor correlations (FC), and error variances (EV) are freed to be different across the two groups. 
normal-weight women who experience these feelings or older women who are experiencing difficulties with controlling their weight.

The second factor, called an anorexia factor, was associated with having problems with low body weight, weight loss, and suffering from anorexia nervosa or an eating disorder. This group did actually have a significantly lower BMI. The bulimia factor, the third factor, involved having suffered from or been treated for bulimia or an eating disorder, having problems with binging and using vomiting as a means of weight control. These two factors are similar to two of the three factors found by Lavik et al. (1991).

The last two factors suggested by the exploratory factor analysis are two purging or weight loss factors. The first factor is associated with the more extreme methods of weight loss, namely starvation, excessive exercise, laxatives, and vomiting. The second purging factor is associated with more socially acceptable methods of weight loss, namely fluid and slimming tablets. These two factors seem to be associated with different types of women. Younger women who are more educated and earning a larger income tend to use the extreme weight loss methods, whilst overweight women with less education use the milder forms of tablet purging. Previous studies have not measured methods of weight loss, with the exception of dieting or cutting back on food intake. Such dietary restraint is a common behavior practiced by many women at some stage in their life. Nevertheless, the other methods of weight control, though less commonly practiced, need to be investigated in their own right. This finding emphasizes the need to include a variety of measures of purging and weight loss in future studies of disordered eating in the general community, as they appear to form significant groups of behavior.

\section{Robustness of Eating Structure}

Whilst exploratory factor analysis is a commonly used tool of investigation of latent factors underlying observed variables, the use of CFA is less common and the use of cross-validation to test generalizability is almost nonexistent (Marsh, 1991). This study has been able to use all these methods to provide some evidence of the robustness of the factor structure. The use of CFA suggests that the model is close to being adequate and requires only a few additional parameters (in the form of factor loadings) to produce a highly acceptable model. Using both the Tucker's congruency coefficient and the availability of Twin 1 (first in birth order) and Twin 2 (second in birth order) groups to examine generalizability of the factor structure, it appears that each factor is basically the same across the groups. However, some caution must be used when attempting to generalize these findings to other populations as the factor correlations between the groups differ somewhat, particularly in relation to the bulimia and first purging factor. This may indicate that these two factors are not independent and may appear as one factor in another population, perhaps a general bulimia nervosa factor.

\section{CONCLUSIONS}

In conclusion, we suggest that where disordered eating exists in the female community, it is possible to differentiate five different types of disordered eating behaviors. There are women who are overweight and have some problems with controlling their weight, women who are underweight and suffering from anorexia nervosa or some 
eating disorder not otherwise specified in this sort of area, women who have problems with bulimic behavior, women who use extreme methods of weight control, and a group of overweight women who use slimming or fluid tablets to attempt to control weight. Future research in this area needs to ensure that the different methods of purging are included in assessments. It would be of great interest to further investigate these clusters of behaviors and their relationship to measures of individual psychopathology, personality, and perceptions of family.

This work was supported by grants from the National Health and Medical Research Council (Australia) and National Institutes of Health (AA07535) (U.S.A.) to A.C.H. and N.G.M. and a Flinders URB grant to T.W. and M.T. We thank Olivia Zheng for data entry, John Pearson for data management, and David Duffy and Sue Taylor for help with computing Tucker's congruency coefficients. We also thank the twins for their cooperation.

\section{REFERENCES}

American Psychiatric Association (1980). Diagnostic and statistical manual of mental disorders. (3rd ed.). Washington, DC: Author.

American Psychiatric Association (1987). Diagnostic and statistical manual of mental disorders. (3rd rev. ed.). Washington, DC: Author.

American Psychiatric Association (1994). Diagnostic and statistical manual of mental disorders. (4th ed.). Washington, DC: Author.

Ben-Tovim, D. I., \& Walker, M. K. (1992). A quantitative study of body-related attitudes in patients with anorexia and bulimia nervosa. Psychological Medicine, 22, 961-969.

Bentler, P. M. (1990). Comparative fit indexes in structural models. Psychological Bulletin, 107, 238-246.

Bentler, P. M., \& Bonett, D. G. (1980). Significance tests and goodness of fit in the analysis of covariance structures. Psychological Bulletin, 88, 588-606.

Coker, S., \& Roger, D. (1990). The construction and preliminary validation of a scale for measuring eating disorders. Journal of Psychosomatic Research, 34, 223-231.

Feldman, L. A. (1993). Distinguishing depression and anxiety in self-report: Evidence from confirmatory factor analysis on non-clinical and clinical samples. Journal of Consulting \& Clinical Psychology, 61, 631-638.

Ferguson, E., Dodds, A., Ng, L., \& Flannigan, H. (1994). Perceived control: Distinct but related levels of analysis. Personality and Individual Differences, 16, 425-432.

Heath, A. C., Cloninger, C. R., \& Martin, N. G. (1994). Testing a model for the genetic structure of personality: A comparison of the personality systems of Cloninger and Eysenck. Journal of Personality and Social Psychology, 66, 762-775.

Joreskog, K. G., \& Sorbom, D. (1993). Lisrel 8: Structural equation modelling with the SIMPLIS command language. Chicago: Scientific Software International, Inc.

Lavik, N. J., Clausen, S. E., \& Pedersen, W. (1991). Eating behaviour, drug use, psychopathology and parental bonding in adolescents in Norway. Acta Psychiatrica Scandinavia, 84, 387-390.

MacCallum, R. (1986). Specification searches in covariance structure modelling. Psychological Bulletin, 100, 107-120.

Marsh, H. W. (1990). Confirmatory factor analysis of multitrait-multimethod data: The construct validation of multidimensional self-concept responses. Journal of Personality, 58, 661-692.

Marsh, H. W. (1991). Multidimensional student's evaluations of teaching effectiveness: A test of alternative higher-order structures. Journal of Educational Psychology, 83, 285-296.

Marsh, H. W., \& Smith, D. (1987). Cross-national study of the structure and level of multidimensional selfconcepts: An application of confirmatory factor analysis. Australian Journal of Psychology, 39, 61-77.

Mehrabian, A., \& Riccioni, M. (1986). Measures of eating-related characteristics for the general population: Relationships with temperament. Journal of Personality Assessment, 50, 610-629.

Tobin, D. L., Holroyd, K. A., Reynolds, R. V., \& Wigal, J. K. (1989). The hierarchical factor structure of the coping strategies inventory. Cognitive Therapy and Research, 13, 343-361.

Treloar, S. A. (1992). Genetic and environmental influences on gynaecopsychiatric conditions. Unpublished doctoral dissertation, University of Queensiand.

Tucker, L. R. (1951). A method for synthesis of factor analysis studies (Personnel Research Section Rep. No. 984). Washington, DC: Department of the Army.

Tucker, L. R., \& Lewis, C. (1973). A reliability coefficient for maximum likelihood factor analysis. Psychometrika, 38, 1-10.

Wade, T., Treloar, S., Abraham, S., Heath, A. C., Martin, N. G., \& Tiggemann, M. (1994). Assessing the epidemiology of bulimia neroosa: Is there a 'best' way? Manuscript submitted for publication. 